\title{
Application of the Questionnaire Method and Fitness Test Method of Modern Physical Education and Training
}

\author{
Wei Wang \\ Wuchang Institute of Technology, Wuhan, 430065, China
}

Keywords: Questionnaire, Physical Fitness Test, Physical Education, Application, Teaching Mode.

\begin{abstract}
Physical education and training discipline at present too saturated, uneven levels of development, lack of the character and other issues. Future development focus to the service at the university sports direction shifted gradually to provide scientific and technological support from conventional monoculture physical education talents for competitive sports were transformed; the perfection of its own system, and comprehensive sports and leisure inside the national traditional sports content on external integration, to continue to absorb the essence of the concept and the other social sciences, natural sciences and meanwhile physical education and training discipline should maintain its own relative independence. We use literature, visit investigation, logical analysis and other research methods, on the existing physical education and training postgraduate course system to investigate, analyze and compare multiple layers. By analyzing China's current physical education and training graduate course system characteristics and problems, we propose to build the basic elements and principled framework training sports master graduate educational curriculum system.
\end{abstract}

\section{Introduction}

With the development of computer and network as the core of modern technology, mankind has quietly entered the information age. And information technology is increasingly transforming our production, lifestyles, ways of working and learning. The increasingly rapid pace of modern education, modern information technology has entered the gate, into the classroom. Various disciplines in varying degrees, the use of modern information technology in education, in order to better enhance the quality of teaching. The physical education is a set of knowledge transfer, skills development and physical exercise as one of the diversified disciplines. IT for physical education and training is an essential tool indispensable.

Sustainability is a subject of the discipline is an important foundation for the future ability to adapt to the era of the environment. Physical Education and Training is a sports science disciplines under a branch, but also the basic disciplines of Physical Education. Since the establishment of discipline Physical Education and Training for the development of physical education and even culture of sports talents have played a key role and subject only to the development of continuous improvement, in order to adapt to the country's sustainable development strategy. According to the overall strategy of the future development of Chinese sports and future direction of the overall development of various disciplines, proposed the development trend of physical education and training disciplines.

With the progress of society, the educated education requirements are increasing, how to make science teaching more fully pass on to the educated, it is a relentless pursuit of the goal of today's educators, and the development of technology for the trainer with to a great convenience, such as the development and application of the multimedia, video, audio, three modeling and other modern technology, the content of sports training and teaching situation of organic integration, to enable students to produce a sense of immersive, infection get inspiration and inspiration. Students produced in a particular context of the strong interest and desire for knowledge, making an already boring hard sports training becomes fun to effectively mobilize the initiative and indomitable will of the quality of students. In addition, teachers can use modern information technology to record yourself or take some of the relevant competitions, training clips, or stream these videos during sports training courseware, so that can mobilize the enthusiasm of the students, and to make some technical movements more intuitive, vivid, so stimulate student interest in the learning to improve teaching effectiveness. Use of 
information technology, to establish the correct visual appearance of movement, can effectively improve sports skills. In the multi-channel audio-visual media provide information to adjust temporal changes in visual phenomenon and a true reflection of the things sports variation terms, significantly better than the purely visual and auditory media. In training at the same time mobilize audiovisual features that can significantly improve the efficiency of learning and development capabilities.

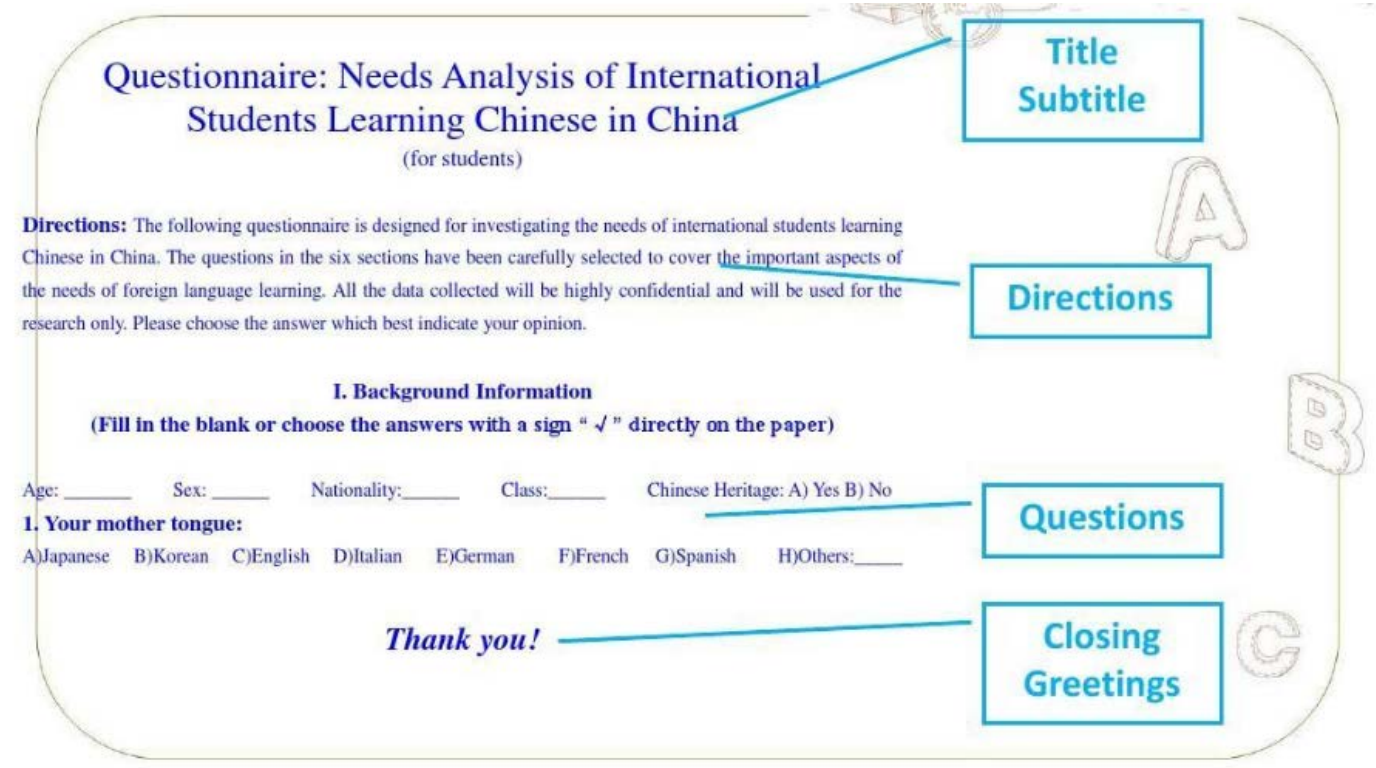

Figure 1. Structure of A Qusetionnaire

In the actual sports training process, some sports technology not only sports complex, and requires a snap. Taekwondo as 360 degrees whirlwind leg kick action, fast pace of exchange in which the feet, waist hip big leg and cooperation and other actions, are very rare in daily life sports action, which undoubtedly bring to teaching and training very difficult. Although this teacher repeatedly explained, many demonstrations, but there are still many students to action and change the timing of the completion of the general process not clear, not essentials, so that teaching effectiveness can not be guaranteed. One teacher's demonstration action subject to many restrictions, arbitrary large; the other students viewing angle and timing is very much restricted, due to the action fleeting, comprehensive high degree of difficulty, it is difficult to clearly observe how to teachers according to the operational requirements for the demonstration, which will inevitably bring some impact to the learning and training of the object. Use of the information technology to a variety of sports technology, technical difficulties, the focus, making common malfunction or surface slides courseware, during training allow students to watch, compare and analyze together with the students, ask questions, answer questions, can promote students analytical problem solving skills, training may help to target faster and more complete representation of the action to establish and improve the awareness stage action learning efficiency in a relatively short period of time to improve motor skills

\section{The Proposed Methodology}

\subsection{Trend of Physical Education and Training Discipline.}

Since China has experienced a successful Olympic Games, the state and the people already on our existing athletes training mechanism has been re-examine and propose a strategic approach by the sports power to a sports power forward. The core of this strategy is the construction of the sports power to change the way athletes train and mechanisms. In the past, our main disciplines Physical Education and the Training for Physical Education teachers, coaches, physical education and training staff and setting arrangements. With colleges and universities to carry out high-level athlete incubation strategy, the future development of Physical Education and Training subjects tend to assist in training high-level athletes. At the forefront of Tsinghua University, Tsinghua University and the 
Institute of Physical Education in itself do not have Physical Education Department, but there is master and doctoral Physical Education Training. Physical Education and Training disciplines is perfect, not only trained a large number of scientific research, education and sports talent, but also for training Tsinghua high-level athletes provide a strong support, Tsinghua University, in a few year time trained college athletes and other international competitions a number of truly competitive for the overall development of China's track and field career to play a crucial role. Tsinghua University Tsinghua major sports training disciplines and provide a support base PE specialized technical training in school athletes. Most studies of Tsinghua University Physical Education and Training Discipline Students are converted to improve athletic performance above. Tsinghua University's success is called the "Tsinghua model", this mode is also bound to the future of our culture called benchmarking Competitive Athletes. Success is inseparable from Tsinghua University Tsinghua mode support discipline Physical Education and Training, and the "Tsinghua model" is also one of the most significant "results" Physical Education and Training Disciplines. Tsinghua's success to China's culture of competitive sports experts also pointed out the direction, but also the future development trend of discipline Physical Education and Training provides a new guide: it is for the campus to carry out competitive sports to provide support.

\subsection{IT technology in motion diagnosis, analysis function.}

Application of Information Technology in Sport Training technical diagnostics and technical analysis is more and more widely used, in which the position has become increasingly important as information technology enables the movement of technical diagnostics, analysis more accurate, more conducive to improve athletic performance training. In the traditional university sports training to improve skills of the student movement relies mainly on teachers and students own experience. In order to analyze one's own and other students' strengths or weaknesses, the students can also use the camera movements into a movie, and then show slow motion shots for people to analyze. However, this is difficult to quantify, rapid, comprehensive and accurate analysis. When the advanced computing, high-speed cameras and other high-tech electronic sensing technology into the field of sports, this situation would fundamentally change occurred. US track and field events in the development of applied computer technology diagnostic systems, high-speed video recorder can be photographed missiles shot trajectory and status within a few seconds, at any time showing acceleration, angle velocity, angular velocity and other motion parameters, and optimizing data can compare the actual parameter and memory, in a timely manner to improve the students' attitude when throwing, hand movements, and thus will enhance the athletic performance training.

Information technology, computer-aided system of sports training sports training and computer applications will further combine the use of computer acquisition, storage and analysis of training data to help teachers manage daily training information, training aid decisions and motion simulation results. This is an important part of the training process to achieve full control of the movement. Computer-aided training through sports crossover study of the sports training, sports medicine, biomechanics and information science and other disciplines, to improve training efficiency and level of training, experience to avoid the traditional reliance training deficiencies and in support of the computer-assisted exercise training system, the teachers can pay more attention to training for the characteristics of individual students, so as to avoid high levels of student achievement appear long stagnation phenomenon. With the development of computer technology, computer sports training system will play a greater role in the sport and the overall goal of making it the best obedience training systems in order to achieve optimal level, greatly improving the effectiveness of training sports training. In the sports field, in order to create the ideal athletic performance, except scientific and rational, systematic and effective way of training methods, a serious scientific means and methods of selection of the elite athletes and sports adapted to the very important, therefore Scientific Selection It will undoubtedly become an important means of Physical training. Rational and effective use of modern information technology, combined with biochemistry, genetics, mathematics and biomechanics and biotechnology, quantitatively oriented model to establish the desired objectives, the development of scientific training object selection criteria, and achieving the Scientific Selection. 


\subsection{Application of Information Technology on Students in Physical Training.}

They can efficiently various teaching the message text, images, sound and animation and other organic combination together, without time and space constraints, communicated directly to the educated sensory organs, thus achieving Education in Physical Training efficient transmission of information, help students take the initiative to engage in observation, experimentation, conjecture, creation (modeling), verification, reasoning, communication and problem-solving activities, help to change the way students learn, it is conducive to the implementation of the dominant position of students, help changes in teaching roles, conducive to building a new type of teacher-student relationship.

Information technology, multimedia computer-assisted teaching physical education and training as a means of optimizing modern sports training teaching to stimulate students' interest in sports training and motivation, enhance the students the concept of action sports and theoretical knowledge to improve the quality of teaching PE, with a very significance and role. Teachers can realistically reflect visually through images, animation, video, audio and other media dynamic process technology operation, to attract students of auditory and visual induce students 'thinking, to achieve the purpose to stimulate students' motivation to learn of.

\section{Conclusion}

Application of information technology in physical education and training will become increasingly widespread, which for us sports educator is both an opportunity and a challenge. How to use new technological means of the creative education and training to improve the current level of sport is an important issue that we think long-term. Currently postgraduate courses and training program of Physical Education and Training curriculum is based on two subjects, resulting in a more specialized postgraduate knowledge structure is not conducive to cultivate general cross-disciplinary graduate, recommended by the principle of wide caliber, reaching press disciplines foundation, two subjects were cultured, and accordingly offer internet courses and orientation courses. Physical training research currently there are two directions of education curriculum model, which should reflect the general trend to broaden the coverage of the professional, and efforts to maintain and play to the characteristics and advantages of the original. Master of physical education and training graduate curriculum system stressing curriculum frontier and multidisciplinary while focusing on knowledge structure and ability structure curriculum system, highlighting the curriculum personalization, which is to construct a reasonable master of physical education and training graduate the basic principles of curriculum system, but also to cultivate high quality innovative talents important guarantee.

\section{References}

[1] He, Zhonghui, and Guangan Hao. "Physical Education Reform and Development in Regular Institutions of Higher Learning under the Vision of Modern Great Education." Advances in Physical Education 4.01 (2014): 36.

[2] Zhang, Xin. "Research of Modern Physical Education Technology Based on Artificial Intelligence." Proceedings of the 2012 International Conference on Cybernetics and Informatics. Springer New York, 2014.

[3] Qiu, Jin. "Application Research on "Seminar" Teaching Method of "Outline of Modern Chinese History"." (2015).

[4] Marcus, Melissa. "A Comparison of Physiological and Psychological Characteristics among Sport Baton Twirlers, Competitive Cheerleaders, and Modern Dancers." (2014). 
[5] Semrad, Alexandra. "Modern secondary education and economic performance: the introduction of the Gewerbeschule and Realschule in nineteenth-century Bavaria." The Economic History Review 68.4 (2015): 1306-1338. 